University of Nebraska - Lincoln

DigitalCommons@University of Nebraska - Lincoln

CSE Conference and Workshop Papers

Computer Science and Engineering, Department

2006

\title{
Autonomous Clustering-Based Heterogeneous Waveband Switching in WDM Networks
}

\author{
Mengke Li \\ University of Nebraska-Lincoln, mli@cse.unl.edu \\ Byrav Ramamurthy \\ University of Nebraska-Lincoln, bramamurthy2@unl.edu
}

Follow this and additional works at: https://digitalcommons.unl.edu/cseconfwork

Part of the Computer Sciences Commons

Li, Mengke and Ramamurthy, Byrav, "Autonomous Clustering-Based Heterogeneous Waveband Switching in WDM Networks" (2006). CSE Conference and Workshop Papers. 73.

https://digitalcommons.unl.edu/cseconfwork/73

This Article is brought to you for free and open access by the Computer Science and Engineering, Department of at DigitalCommons@University of Nebraska - Lincoln. It has been accepted for inclusion in CSE Conference and Workshop Papers by an authorized administrator of DigitalCommons@University of Nebraska - Lincoln. 


\title{
Autonomous Clustering-Based Heterogeneous Waveband Switching in WDM Networks
}

\author{
Mengke Li and Byrav Ramamurthy \\ Department of Computer Science and Engineering, University of Nebraska-Lincoln, USA \\ Email: \{mli, byrav\}@cse.unl.edu
}

\begin{abstract}
Employing waveband switching (WBS) in WDM networks can reduce the network operational cost and the call blocking probability. However, upgrading the existing optical switching architecture requires time and money. It is expected that a heterogeneous waveband switching (HeteroWBS) architecture would be desirable, where some nodes can support WBS functions and some cannot. We study the performance of HeteroWBS networks in terms of call blocking probability and cost savings under dynamic traffic requests. We propose an autonomous clustering-based HeteroWBS (AS-HeteroWBS) architecture to clusters the network into multiple autonomous systems (ASs). An AS may contain some specific nodes that provide WBS functions for all the nodes in the AS. Based on the architecture, three HeteroWBS algorithms are proposed. Our simulation results show that the HeteroWBS algorithms can achieve optimal cost savings while maintaining the same network throughput compared with the algorithm without WBS.
\end{abstract}

\section{INTRODUCTION}

Wavelength division multiplexing (WDM) is an important technique for the next-generation networks [1], [2]. As the number of wavelengths in a fiber increases, the WDM network provisioning and management functions become more complex and the number of optical components increases. Waveband switching (WBS) has been proposed as an efficient solution in [3]-[5] to solve those problems.

Through WBS, a small set of wavelengths is aggregated into a waveband (wavelength band) at an optical crossconnect (OXC), transmitted as a whole along fiber links, and disaggregated back into wavelengths at another OXC. A waveband-route, formed along those fiber links and OXCs, can reduce the number of utilized OXC ports compared with the corresponding wavelength-routes (lightpaths) [6]. Thus, adopting WBS can generate more revenue by minimizing the switching and transmission costs in the optical domain of WDM networks, which are dominated by the number of OXC ports used. Other advantages can also be achieved, such as reducing the OXC size and complexity [7].

By adding a waveband crossconnect fabric and adopting mixed band (de)multiplexers, an OXC is capable of waveband switching, which is denoted as band-switching optical cross-connect (BOXC). By adding waveband (de)multiplexers, a BOXC can group and disaggregate wavebands, which is denoted as a multi-granular optical cross-connect (MGOXC) and is shown in Fig. 1. An MGOXC can also adopt (de)multiplexers that (de)multiplex wavebands from a fiber first and then (de)multiplex wavelengths from wavebands. Such an MGOXC is illustrated in [3], [5].
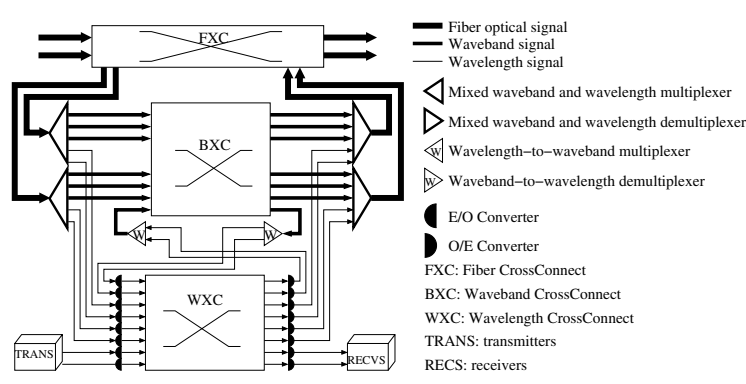

Fig. 1. An illustration of a multi-granular optical cross-connect (MGOXC) capable of mixed waveband/wavelength switching, wavebands grouping, and wavebands disaggregating.

A WDM network with the functionality of WBS is a waveband-switching network (WBS network). However, upgrading the existing optical switching architecture requires time and money. It is expected that a heterogeneous waveband switching (HeteroWBS) architecture would be desirable, where some nodes can support WBS functions and some cannot. As the blocked calls influence the network throughput, it is important to study the performance of HeteroWBS networks in terms of call blocking probability and cost savings.

In a HeteroWBS network, dynamically arriving traffic should be provisioned through either wavelength-routes or waveband-routes. We analyze the HeteroWBS network architecture and cluster the whole network into multiple autonomous systems (ASs). Based on the autonomous clustering architecture, three HeteroWBS algorithms adopting different routing algorithms are proposed. Substantial simulations are conducted to study the network performance. Our simulation results show that the HeteroWBS algorithms can achieve optimal performance compared with the algorithm without waveband switching.

The rest of the paper is organized as follows. Section II defines the dynamic HeteroWBS problem in detail. Section III describes the autonomous clustering-based HeteroWBS architecture. Section IV presents the three algorithms based on the architecture. The simulation results are illustrated in Section V. Finally, Section VI concludes the paper.

\section{Network Configuration And Problem STATEMENT}

\section{A. Network Node Architecture}

We consider the mixed waveband-wavelength switching, where the (de)multiplexing of wavebands and wavelengths 
from a fiber is completed at the same time by adopting band (de)multiplexers. Fig. 1 presents the architecture of such an MGOXC. An MGOXC may support two types of waveband switching, namely the uniform waveband switching (UWBS) and the non-uniform waveband switching (NUWBS). In both types of waveband switchings, the waveband granularity is an important factor in achieving maximum cost savings [5], [8]. In this paper, we consider UWBS.

\section{B. Network Configuration}

In a HeteroWBS network, there are two types of network nodes. Some are MGOXCs supporting WBS functions. The others are ordinary OXCs. The network can be divided into two hierarchies, namely the wavelength crossconnecting (WXC) layer and the waveband crossconnecting (BXC) layer. Establishing and tearing down connections may involve nodes of both types through both layers.

Let the mesh network consist of $N$ nodes and $J$ unidirectional links. Each node is a routing node with an MGOXC or an OXC. Each link is a fiber link carrying $W$ wavelengths. It may also carry $B$ wavebands, each of which may consist of at most $G$ specific wavelengths. $G$ is also referred to as the waveband granularity. For a HeteroWBS network supporting mixed waveband-wavelength switching, only a fraction of wavelengths are grouped in wavebands. Therefore, a fiber link may contain both active wavelengths and active wavebands and $B \times G \leq W$. We define a ratio $r=\frac{B \times G}{W}$, where $0 \leq r \leq 1 . r$ is the ratio of the number of wavelengths in wavebands to the total number of wavelengths in a fiber. In addition, depending on the utilized MGOXCs, the set of wavelengths in a waveband is specified beforehand. For example, a waveband $b_{i}$ may consist of $G$ contiguous wavelengths $w_{(i-1) G}, w_{(i-1) G+1}, \ldots, w_{i G-1}$.

\section{Problem Statement and Connection Management}

In a HeteroWBS network, a dynamically arriving call should be provisioned either along a wavelength-route or along an intermediate/end-to-end waveband-route [9]. The problem of provisioning a call in a HeteroWBS network can be divided into searching for a candidate path, referred to as routing, searching for a free wavelength with or without waveband grouping requirements, referred to as wavelength assignment, and searching for an available waveband along the sub-paths of the candidate path, referred to as waveband assignment. The whole problem is then called routing, wavelength and waveband assignment (RWWBA) problem. To focus on adopting the WBS technique in a HeteroWBS network, it is assumed that each call requires a whole wavelength capacity. We also conducted simulations in networks, where each call may require more than one wavelength. The results are similar.

A connection in a HeteroWBS network may be originated from and terminated at either an OXC or an MGOXC. To accommodate the connection, a waveband-route may be set up or adopted along a sub-path. All the nodes along the waveband-route should be MGOXC nodes. As a wavebandroute should be at least two hops to be cost-efficient [4], the path for a connection through multiple intermediate wavebandroutes has at least four hops. In addition, the available wavelengths are restricted to those in the waveband along all the waveband-routes and along the remaining wavelengthroutes. Moreover, provisioning connections through multiple intermediate waveband-routes may increase the path length unnecessarily. This may waste the network resources, which in turn increases the call blocking probability and degrades the network performance. Thus, a connection is allowed to be routed through at most one intermediate waveband-route.

\section{Autonomous Clustering-Based HeterogeneOUS WAVEBAND-SWITCHING ARCHITECTURE}

To manage the connections, the HeteroWBS network is clustered into multiple autonomous systems (ASs) ruled by the grouping strategy. The most prominent characteristic of an $\mathrm{AS}$ is that the WBS managements are controlled only by the MGOXC nodes in the AS. This section first presents an efficient autonomous connection management, which can conceal the WBS information from the ordinary OXC nodes. To support the efficient autonomous connection management, an autonomous clustering-based heterogeneous waveband switching (AS-HeteroWBS) architecture is then proposed.

\section{A. Autonomous Connection Management}

When a connection request is initiated by the end-users or the upper layer applications, the source node searches for an available path. In doing so, the nodes communicate with each other about their current configurations. As MGOXC nodes are responsible for managing waveband switching, the information about the waveband availability and the wavelength availability in the wavebands should be shared among the MGOXC nodes. However, the type of information is not necessary for the ordinary $\mathrm{OXC}$ nodes. The communication among the ordinary $\mathrm{OXC}$ nodes for the type of information is not necessary. Thus, we introduce autonomous connection management to discover the current network configurations while screening the WBS information from the ordinary OXC nodes.

It is important to point out that the MGOXC nodes should be connected with other MGOXC nodes to take advantage of WBS. Initially, an MGOXC node does not know which of its neighbors are MGOXC nodes. An initial BXC message should be distributed. If its ordinary OXC neighbor receives this message, it simply discards it. Otherwise, upon receiving the message, the neighbor MGOXC node should response with its available wavebands and available wavelengths in the wavebands. Additionally, to discover the initial network configuration, all MGOXCs and ordinary OXCs distribute an initial WXC message and confirm the presence of their neighbors with their available wavelengths upon the receipt of the message.

A connection originating from an ordinary OXC may go through a waveband-route. However, the source node has no information about the current available wavebands. It should 
communicate with one of the MGOXCs in the network. Moreover, if the MGOXC is along the waveband-route, it would eliminate the waveband-route information and just advise the source node with the available wavelengths in wavebands. Through this method, the waveband switching is transparent to the ordinary OXCs. Based on the idea, we propose an autonomous clustering-based HeteroWBS architecture in the following subsection.

\section{B. Autonomous Clustering-Based Heterogeneous WBS Archi- tecture}

An autonomous clustering-based heterogeneous waveband switching (AS-HeteroWBS) optical network architecture is proposed to make WBS transparent to the ordinary OXCs. The clustering of the network is ruled by the grouping strategy. First, the grouping strategy attaches MGOXCs to ordinary OXCs in the network. Given a HeteroWBS network with a set of connected MGOXCs, an ordinary OXC $n_{c}$ selects its attached MGOXCs according to the following.

- If $n_{c}$ has neighbor MGOXCs, it adds all the neighbor MGOXCs as its attached MGOXCs. Otherwise, continue.

- If $n_{c}$ can reach multiple MGOXCs that are two-hops away, it picks the one with the smallest distance as its attached MGOXC. Otherwise, continue.

- If $n_{c}$ can reach MGOXCs through at least three hops, it will not be assigned with any attached MGOXC. In other words, the connection originating from $n_{c}$ will always be accommodated through a lightpath.

The rationale of assigning an attached MGOXC that is one-hop or two-hops away to an OXC is to adopt WBS for a connection with maximum possibility. Moreover, by not attaching MGOXCs that are at least three-hops away from the OXC node, we can reduce the possibility of wasting network resources in routing connections through long paths along intermediate waveband-routes.

After determining the attached $\mathrm{MGOXC}(\mathrm{s})$ for each ordinary $\mathrm{OXC}$, the ASs can be determined as follows.

- If the ordinary OXC nodes $n_{i 1}, . . n_{i j}$ have the same attached MGOXC node $b_{k}$ and $b_{k}$ is the only attached MGOXC for all of them, the ordinary nodes $n_{i 1}, . . n_{i j}$ and the MGOXC $b_{k}$ are clustered into an AS $\left\{n_{i 1}, . . n_{i j}, b_{k}\right\}$.

- Assume that an ordinary OXC node $n_{i}$ has only one attached MGOXC $b_{j 1}$ and $b_{j 1}$ is one of the attached MGOXC in an AS $\left\{n_{k 1}, . ., n_{k x}, b_{j 1}, . ., b_{j y}\right\}$. If $n_{i}$ is at most two-hops away from all of the attached MGOXCs $b_{j 1}, . ., b_{j y}$ in the AS, add $n_{i}$ into the AS. Otherwise, $n_{i}$ and $b_{j 1}$ are clustered into a separate AS.

- If there is no attached MGOXC for an ordinary OXC $n_{i}$, it is clustered into a separate AS, which contains node $n_{i}$ only.

The clustering process continues until there is no change for all the ASs in the network. The rationale of enlarging the ASs is to encourage the adoption of lightpaths with only one or two hops.

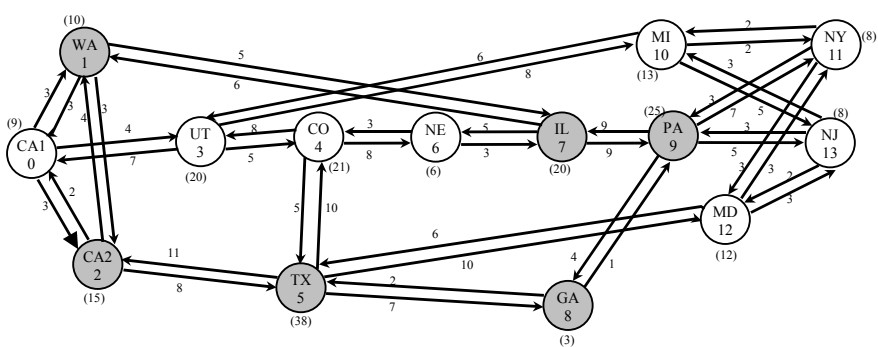

(a) The heterogeneous NSF network, where gray nodes are MGOXC nodes and white nodes are ordinary $\mathrm{OXC}$ nodes

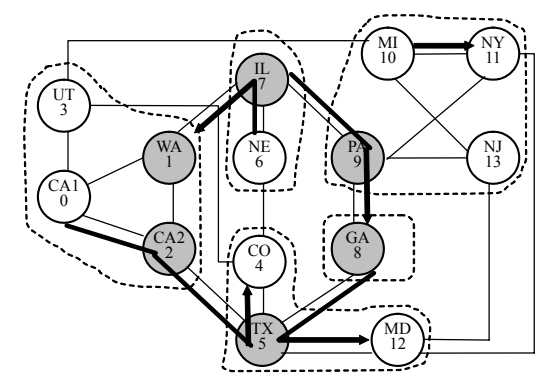

(b) The clustering NSF network. The dash circle delimitates the cluster and the gray nodes are the group nodes in the cluster.

Fig. 2. An illustration of clustering the nodes in a heterogeneous waveband switching network.

An illustration of clustering the HeteroWBS network into ASs is shown in Fig. 2. The example NSF network is a HeteroWBS network with six MGOXC nodes, which are $W A_{1}$, $C A 2_{2}, T X_{5}, I L_{7}, G A_{8}$, and $P A_{9}$. The ordinary OXC node $U T_{3}$ can reach three MGOXCs through two-hops routes. The three MGOXCs are $W A_{1}, C A 2_{2}$, and $T X_{5}$. Node $U T_{3}$ selects MGOXC $W A_{1}$ as its attached node since the path from $U T_{3}$ to $W A_{1}$ is the shortest. Initially, node $C A 1_{0}$ selects all the neighbor MGOXCs $C A 2_{2}$ and $W A_{1}$ as its attached MGOXCs. Since $U T_{3}$ is only two-hops away from $C A 2_{2}$, the clustering process adds $U T_{3}$ into the AS $\left\{C A 1_{0}, C A 2_{2}, W A_{1}\right\}$. The other ASs can be derived through the same process and are shown in Fig. 2. If the OXC has no attached MGOXC, it by itself forms an AS. The MGOXC nodes in the ASs are called the group nodes.

\section{Heterogeneous Waveband Switching ALGORITHM DESIGN}

Three HeteroWBS algorithms are proposed based on the AS-HeteroWBS architecture to solve the dynamic RWWBA problem. The algorithms adopt first-fit waveband assignment and first-fit wavelength assignment. We focus on the HeteroWBS algorithm design with different routing algorithms.

The HeteroWBS algorithms provision a connection request $c(s, d)$ upon its arrival dynamically. $c(s, d)$ can be one of the following eight classes in the AS-HeteroWBS network.

[C1] $s$ and $d$ are within the same AS, where $s$ and $d$ are both MGOXCs.

[C2] $s$ and $d$ are within the same AS, where $s$ is an MGOXC and $d$ is an ordinary OXC. 
[C3] $s$ and $d$ are within the same AS, where $s$ is an ordinary OXC and $d$ is an MGOXC.

[C4] $s$ and $d$ are within the same AS, where $s$ and $d$ are both ordinary OXCs.

[C5] $s$ is in different AS from $d$, where $s$ and $d$ are both MGOXCs.

[C6] $s$ is in different AS from $d$, where $s$ is an MGOXC and $d$ is an ordinary OXC.

[C7] $s$ is in different AS from $d$, where $s$ is an ordinary OXC and $d$ is an MGOXC.

[C8] $s$ is in different AS from $d$, where $s$ and $d$ are both ordinary OXCs.

\section{A. Autonomous Heterogeneous Waveband Switching (AS- WBS) Algorithm}

Now the network nodes have been clustered into multiple ASs. The MGOXCs comprise the BXC layer in the ASHeteroWBS network. We propose the AS-WBS algorithm to manage BXCs only at the group nodes of each ASs. The ASWBS algorithm accommodates connections according to the following three principles.

- A waveband-path is more preferable than a lightpath.

- A lightpath $\left(L_{i}\right)$ across ASs for a connection is preferred if the other paths are at least two hops longer than $L_{i}$. For example, if a connection request $c\left(U T_{3}, C O_{4}\right)$ arrives, the lightpath across ASs along route $U T_{3} \rightarrow \mathrm{CO}_{4}$ is preferred as shown in Fig. 2(b).

- The path with the smallest hop count within an AS is preferred.

We describe the AS-WBS algorithm for a connection request $c(s, d)$ one class at a time as follows.

I. $c(s, d)$ is in class $\mathrm{C} 1$ or $\mathrm{C} 5$ :

- if there is an active waveband-path $B(s, d)$ from $s$ to $d$ with available wavelengths, $c$ is grouped into $B$.

- Otherwise, if a new waveband-path $B_{w}(s, d)$ from $s$ to $d$ along the shortest path can be set up, $c$ is routed through $B_{w}$.

- Otherwise, if a new lightpath $\lambda_{c}$ along the shortest path can be set up, $c$ is routed through $\lambda_{c}$.

- Otherwise, adopt the k-shortest path algorithm and search for any available path.

- If no path is available, $c$ is blocked.

II. $c(s, d)$ is in class $\mathrm{C} 2, \mathrm{C} 3$, or $\mathrm{C} 4$ :

- Recall that a path within the AS is preferred to the one across the AS if the path is short. Thus, AS-WBS searches for an available shortest path within the cluster and sets up a new lightpath if the path is only one or two hops long. Otherwise, continue.

- Adopt the k-shortest path algorithm and search for any available path.

- If no path in available, $c$ is blocked.

III. $c(s, d)$ is in class $\mathrm{C} 6$ :

- Recall that two ordinary OXC nodes will not be clustered together if there is no attached MGOXC for them, even though they are neighbors.

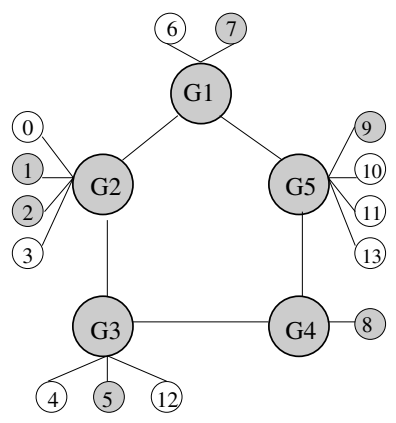

Fig. 3. The GN-network produced from the NSF AS-HeteroWBS architecture, where the big gray circle is the $G$-node with MGOXC nodes, the small gray circle is the MGOXC node, and the small white circle is the OXC node.

- If $s$ is the only node in the AS, adopt the k-shortest path algorithm and search for any available lightpath. If no path is available, $c$ is blocked.

- If in node $s$ 's $\mathrm{AS}$, there is a set of MGOXCs $\left\{B_{i 1}, \ldots, B_{i j}\right\}$ and $j \neq 0$. Search for an active waveband-route from MGOXC node $B_{i x}$ to $d$ with an available wavelength $\lambda$ such that $\lambda$ is also available from s to $B_{i x}$, where $1 \leq x \leq j$.

- Otherwise, try to set up a new shortest wavebandpath from $B_{i y}$ to $d$ with a free wavelength that is also available along the path from $s$ to $B_{i y}$, where $1 \leq y \leq j$.

- Otherwise, adopt the k-shortest path algorithm and search for any available lightpath.

- If no path is available, $c$ is blocked.

IV. $c(s, d)$ is in class $\mathrm{C} 7$ or $\mathrm{C} 8$ :

- The same as for a call in class C6, except that d's AS should be checked instead.

\section{B. Autonomous Source-Limited Heterogeneous Waveband Switching (AS-S-WBS) Algorithm}

The AS-S-WBS algorithm is only different from the ASWBS algorithm in its routing. When a connection request of class $\mathrm{C} 7$ or $\mathrm{C} 8$ arrives, the source node delivers its connection request to the nearest attached $\mathrm{MGOXC}$ in the AS. After the MGOXC receives the connection request, it searches for the shortest path to the destination with a waveband-route along it. It also searches for a shortest-path from the source node to the MGOXC and combines the routes. For a connection request of class $\mathrm{C} 1, \mathrm{C} 2, \mathrm{C} 5$, or $\mathrm{C} 6$, the source node is the MGOXC. For a connection request of class $\mathrm{C} 3$ or $\mathrm{C} 4$, a shortest-path is assigned without any waveband-route.

\section{Shortest-Path-Based Heterogeneous Waveband Switching (SH-WBS) Algorithm}

We propose the SH-WBS algorithm based on the simplification of the AS-HeteroWBS architecture. As we mentioned an MGOXC should be connected with at least one other MGOXC to switch data in optical waveband signals and the MGOXCs comprise the BXC layer in the HeteroWBS network. Thus, we can construct a smaller group-node network (GN-network) 

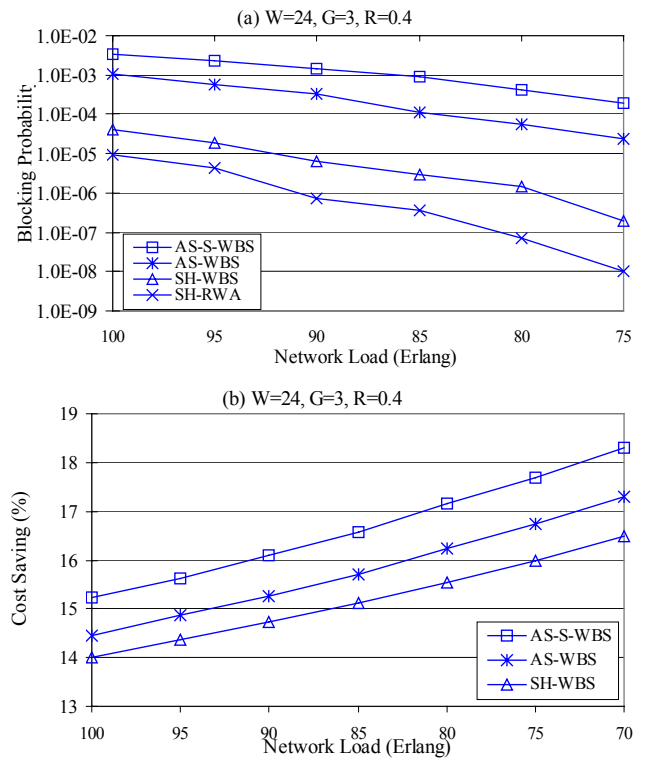

Fig. 4. Comparison of the algorithms under different network traffic loads (L), where $\mathrm{W}$ is $24, \mathrm{G}$ is 3 , and $\mathrm{r}$ is 0.4 . (a) Comparison of the blocking probability. (b) Comparison of the cost saving ratio (\%).

from the AS-HeteroWBS architecture by considering each AS as a single group node (G-node).

There are two types of G-nodes in the GN-network. One is with MGOXC nodes in its AS. The other is without any MGOXC node in its AS. The connectivity of a G-node in the $G N$-network is determined by the connections between the MGOXC nodes in the AS and the MGOXCs in other ASs. If the AS contains only one OXC node, the $G$-node connectivity is determined by the OXC node. The simplified GN-network for the NSF AS-HeteroWBS network is illustrated in Fig. 3, where the G-nodes with MGOXCs in their ASs are illustrated by big gray circles. After the $G N$-network is constructed, the nodes in the AS are connected to the G-node. As shown in Fig. 3 , the MGOXC nodes are illustrated in small gray circles and the ordinary OXC nodes are illustrated in small white circles.

When a connection request of class $\mathrm{C} 5, \mathrm{C} 6, \mathrm{C} 7$, or $\mathrm{C} 8$ arrives, the SH-WBS algorithm searches for the shortest path between the two G-nodes in the GN-network. It then expands the real route in the original network by adopting the shortestpath algorithm. For the connection request of class $\mathrm{C} 1, \mathrm{C} 2$, $\mathrm{C} 3$, or $\mathrm{C} 4$, the SH-WBS algorithm searches for the shortest path in the original AS-HeteroWBS network. The wavelength assignment, waveband assignment, and the setting up of a new waveband-route are the same as the AS-WBS algorithm.

\section{Simulation of Heterogeneous WBS Networks}

We employ the NSF HeteroWBS network with 6 MGOXC nodes and 8 OXC nodes shown in Fig. 2(a). The percentage of MGOXCs in the network is 43\%. The AS-HeteroWBS network architecture is shown in Fig. 2(b). The GN-network is shown in Fig. 3. Poisson traffic is generated for the network with a rate $\lambda$. The traffic is uniformly distributed among the
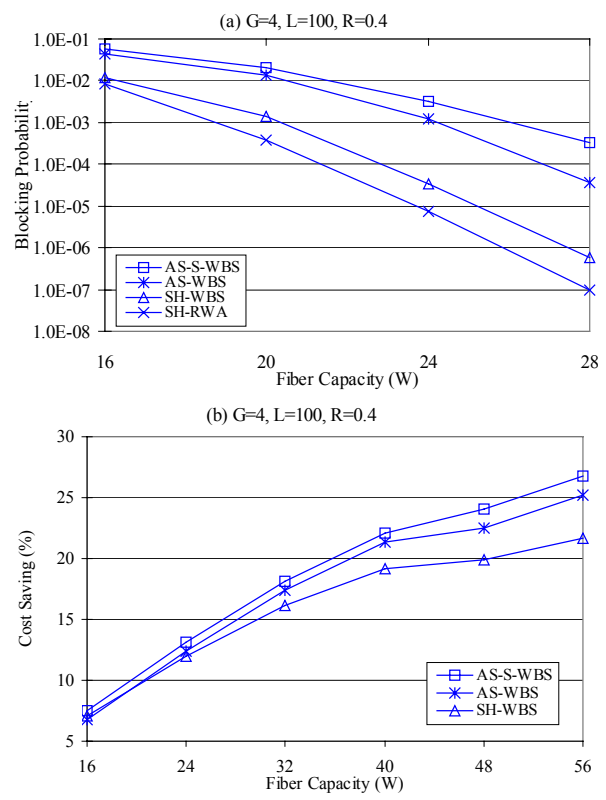

Fig. 5. Comparison of the algorithms under different fiber capacities (W), where $\mathrm{G}$ is 4, $\mathrm{L}$ is 100 Erlang, and $\mathrm{r}$ is 0.4. (a) Comparison of the blocking probability. (b) Comparison of the cost saving ratio (\%).

connection pairs. The call holding time is exponential with a mean time $\frac{1}{\mu}$. The network load in terms of Erlang is $L=\frac{\lambda}{\mu}$.

The following notations are used in this section. $A S_{-} W B S$, $A S_{-} S_{-} W B S$, and $S H_{-} W B S$ represent the results obtained by adopting the AS-WBS algorithm described in Section IV-A, the AS-S-WBS algorithm described in Section IV-B, and the SH-WBS algorithm described in Section IV-C separately. $\mathrm{SH}$ $R W A$ illustrates the results obtained by the RWA algorithm adopting the shortest-path routing and the first-fit wavelength assignment without waveband switching.

We compare the three HeteroWBS algorithms and the RWA algorithm in terms of blocking probability and cost saving ratio. Simulations are conducted under different network loads in terms of Erlang: $L \in\{75,80,85,90,95,100\}$, with different wavelength capacities per fiber link: $W \in$ $\{16,20,24,28,32,36,40,48,56\}$, with different waveband granularities: $G \in\{2,3,4,5,6\}$, and with different ratios of wavelengths in wavebands: $r \in\{0.35,0.4,0.45,0.55,0.65\}$. Under each scenario and for each algorithm, we simulate $100,000,000$ connection requests to obtain the results. The cost savings of a HeteroWBS algorithm are the savings of the utilized ports multiplied with the weighted cost associated with a port. There are two types of ports along a path. One is an $\mathrm{OOO}$ port with a weighted cost 0.2 along wavebandroutes. The other is an OEO port with a weighted cost 1.0 along wavelength-routes. The cost saving ratio is obtained by dividing the cost saving due to WBS by the operational costs without WBS.

Fig. 4(a) compares the algorithms in terms of call blocking probability under different network traffic loads (L). As can be seen, the shortest-path-based HeteroWBS (SH-WBS) 


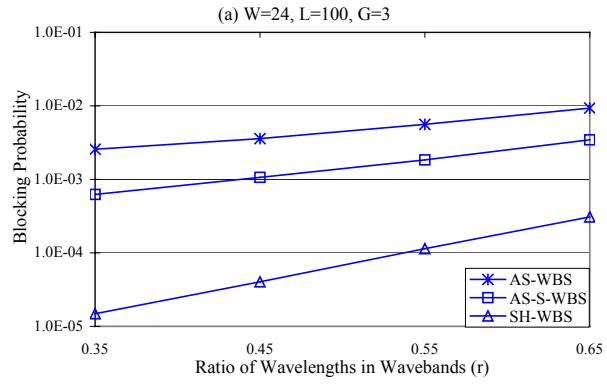

(b) $\mathrm{W}=24, \mathrm{~L}=100, \mathrm{G}=3$

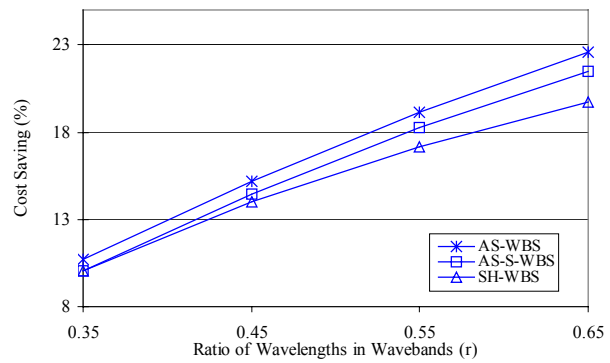

Fig. 6. Comparison of the algorithms under different ratios of wavelengths in wavebands (r), where $\mathrm{W}$ is $24, \mathrm{~L}$ is 100 , and $\mathrm{G}$ is 3 . (a) Comparison of the blocking probability. (b) Comparison of the cost saving ratio (\%).

algorithm can achieve better performance than the other two HeteroWBS algorithms. Moreover, the results of the SH-WBS algorithm are very close to those of the RWA algorithm. By adopting the shortest-path routing only, the average path length for a connection is reduced compared with adopting the kshortest path routing algorithm. Thus, the SH-WBS algorithm consumes fewer resources than the AS-S-WBS and the ASWBS algorithms. In addition, all the results show that the blocking probability decreases as the network traffic load decreases. On the other hand, Fig. 4(b) illustrates that the AS-WBS algorithm and AS-S-WBS algorithm can achieve higher cost saving ratio than the SH-WBS algorithm. This is reasonable as the AS-S-WBS and the AS-WBS algorithms try to utilize WBS as often as possible for a connection. Moreover, the results show that the cost saving ratio increases as the traffic load decreases. The reason is that as the traffic load decreases, the ratio of successfully provisioned connections increases.

Fig. 5(a) compares the algorithms in terms of call blocking probability under different fiber capacities (W). Again, the SHWBS algorithm performs better than the other two HeteroWBS algorithms and is close to the RWA algorithm. Moreover, the results illustrate that the blocking probability decreases as the fiber capacity increases. Meanwhile, Fig. 5(b) illustrates that the AS-WBS and the AS-S-WBS algorithms outperform the SH-WBS algorithm in cost savings. In addition, the results show that the cost saving ratio increases as the fiber capacity increases. This is because as the fiber capacity increases the number of wavebands along a fiber link increases given the fixed $r$ and $G$. Moreover, the higher the fiber capacity is, the larger the performance gap becomes.

Fig. 6(a) compares the call blocking probability and Fig. 6(b) compares the cost saving ratio of the HeteroWBS algorithms when the ratio $r$ changes. Again, the SH-WBS algorithm can achieve lower blocking probability and the ASS-WBS and the AS-WBS algorithms can achieve higher cost savings. Moreover, the blocking probability slightly increases as the ratio $r$ increases. Additionally, the higher the ratio $r$ is, the higher the cost saving ratio that can be obtained. This is because as the ratio $r$ increases the number of wavebands along a fiber link increases given the fixed $W$ and $G$. Thus, the possibility of a call traversing a waveband-route increases.

\section{CONCLUSION}

We investigated the waveband switching (WBS) problem under dynamic traffic requests in a heterogeneous waveband switching (HeteroWBS) network. In such a HeteroWBS network, some nodes are MGOXCs with the WBS functionalities and some are ordinary OXCs without the WBS functionalities. To screen the WBS information from the ordinary OXC nodes, an autonomous clustering-based heterogeneous waveband switching (AS-HeteroWBS) architecture was proposed, where a specific set of OXCs and MGOXCs is grouped into an autonomous system (AS). The MGOXC nodes in an AS are responsible for all the WBS functions in the AS. Three HeteroWBS algorithms were proposed based on the ASHeteroWBS architecture. Substantial simulations showed that the HeteroWBS architecture is efficient to improve the WDM network performance.

\section{REFERENCES}

[1] D. Banerjee and B. Mukherjee, "Wavelength-routed optical networks: linear formulation, resource budgeting tradeoffs, and a reconfiguration study," IEEE/ACM Transcation on Networking, vol. 8, no. 5, pp. 598607 , Oct. 2000.

[2] S. Kaneda, T. Uyematsu, N. Nagatsu, and K. Sato, "Network design and cost optimization for label switched multilayer photonic IP networks," IEEE J. Selected Areas in Communications, vol. 23, no. 8, pp. 1612 1619, Aug. 2005.

[3] L. Noirie, M. Vigoureux, and E. Dotaro, "Impact of intermediate traffic grouping on the dimensioning of multi-granularity optical networks," in Proc. of Optical Fiber Communication (OFC'01]), Anaheim, CA, USA, Mar. 2001, pp. TuG3/1-3.

[4] I. Rauf, G. Samrar, K. Viktor, and C. Aikaterini, "Non-uniform waveband hierarchy in hybrid optical networks," in Proc. IEEE INFOCOM'03, vol. 2, San Francisco, CA, USA, Apr. 2003, pp. 1344-1354.

[5] X. Cao, V. Anand, and C. Qiao, "Multi-layer versus single-layer optical cross-connect architectures for waveband switching," in Proc. IEEE INFOCOM'04, vol. 3, Hong Kong, China, Mar. 2004, pp. 1830-1840.

[6] A. Kolarov and B. Sengupta, "An algorithm for waveband routing and wavelength assignment in hierarchical WDM mesh networks," in Workshop on High Performance Switching and Routing (HPSR'03), Torino, Italy, June 2003, pp. 29-36.

[7] S. Ganguly, N. Tu, R. Izmailov, and H. Mouftah, "Waveband routing and merging in hybrid optical networks," in Proc. IEEE ICC'04, vol. 3, Paris, France, May 2004, pp. 1615-1621.

[8] P. Bullock, C. Ward, and Q. Wang, "Optimizing wavelength grouping granularity for optical add-drop network architecture," in Proc. of Optical Fiber Communication (OFC'03), Atlanta, GA, USA, Mar. 2004, pp. 355357.

[9] M. Li, W. Yao, and B. Ramamurthy, "A novel intermediate waveband switching scheme in WDM mesh networks," in Proc. IEEE GLOBECOM'05, St. Louis, MO, USA, Nov.-Dec. 2005. 